\title{
Deep Impact: excavating comet 9P/Tempel 1
}

\author{
M. F. A'Hearn ${ }^{1}$ and The Deep Impact Team \\ ${ }^{1}$ Department of Astronomy, University of Maryland, College Park MD 20742, USA \\ email: ma@astro.umd.edu
}

\begin{abstract}
The Deep Impact mission delivered 19 gigajoules of kinetic energy to the nucleus of comet 9P/Tempel 1 on 4 July 2005. Intensive observations, both from the two spacecraft and from Earth and Earth-orbit, while approaching the comet led to numerous new findings about comets. Observations of the impact event, from the flyby spacecraft have led us to a determination of numerous physical properties of the nucleus. Analysis of the near-IR spectra is still very preliminary.
\end{abstract}

Keywords. comets: general, comets: 9P/Tempel 1

\section{Introduction}

Deep Impact was conceived to investigate the relationship between the volatiles observed remotely in the ambient coma and ices, presumably primordial, in the bulk of the nucleus. It was also intended to investigate the physical structure of the nucleus itself. The true planning for the mission began in 1995 and the project took 5 years of selling the mission (both to the scientific review panels and to NASA itself) to reach our confirmation to proceed with construction and then an additional 5 years of building the spacecraft and flying the mission. The development of the mission design, the hardware, and the scientific background are described in a series of papers in Space Science Reviews (A'Hearn et al. 2005 and many additional papers cited therein). This IAU Colloquium coincides with the initial submission of the first results to Science (A'Hearn et al. 2005b) and thus this paper, which summarizes content both from the invited review and numerous contributed paeprs, includes only preliminary results corresponding to what is reported there (A'Hearn et al. 2005b). More specifically, we expect that some of the values reported here will change with more analysis. However, we have considered the likely range of errors in drawing conclusions to ensure that all our statements about processes will not change.

\section{The Impact and the Nuclear Properties}

The key goal of the mission was to impact the nucleus with enough energy to excavate to a depth of tens of meters. Whether this would be possible or not depended on the strength of the nuclear material in those outer tens of meters. Experts active in studying cratering had substantial disagreements over whether the formation of the crater would be controlled by the comet's gravity, by the strength of the outer layers, or by compression of porous material with moderate compressive strength, although our own position had been that the formation would likely be controlled by the comet's gravity as discussed by A'Hearn et al. (2005a), by Schultz et al. (2005) and by Richardson et al. (2005). In retrospect, gravitational control is precisely what happened. 


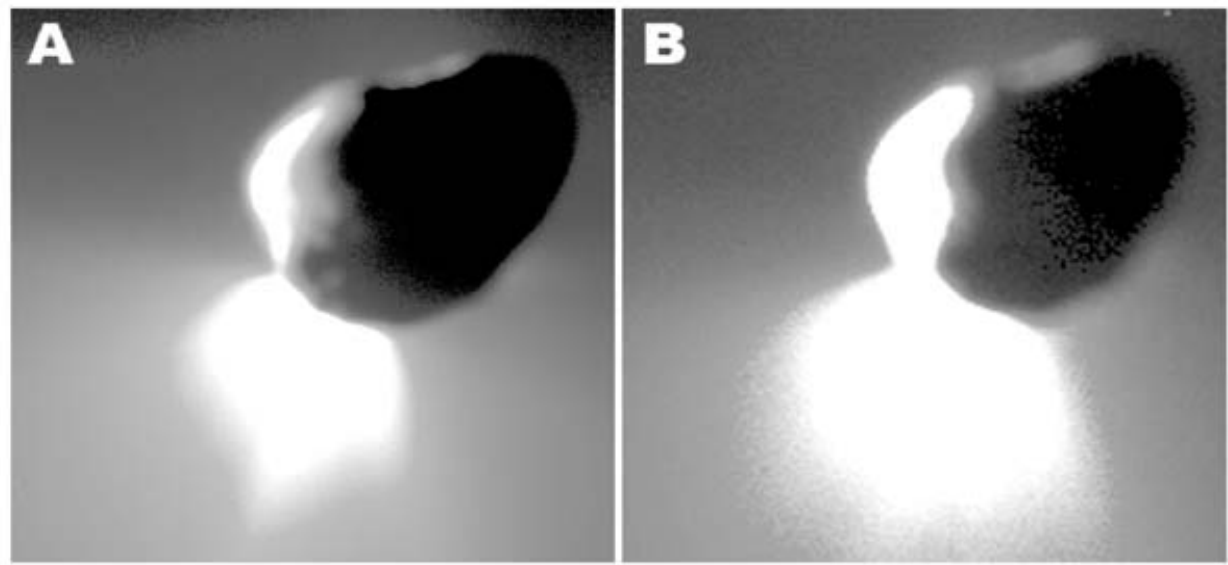

Figure 1. Highly stretched images of comet Tempel 1 taken roughly 45 and 90 minutes respectively after the impact. The ejecta cone has remained attached to the surface and is still expanding at its base as ballistic ejecta fall back to the surface outside the crater.

Our lookback imaging showed that the ejecta cone remained attached to the surface for hours, although strictly speaking we can only say that it remained within a few hundred meters of the surface since the crater itself is behind the limb as seen during lookback imaging. A pair of lookback images, taken at roughly 45 and 90 minutes after impact, is shown in figure 1 and one can easily see the cone still expanding at its lowest level visible around the limb. This is due to ballistic material from the cone falling back to the surface outside the crater and this allows us to estimate both the strength of the material near the rim of the (unseen) crater and the local gravity at the crater. With worst case assumptions, we find that the shear strength near the rim must be no more than $65 \mathrm{~Pa}$, implying remarkably weak material at the rim. For the acceleration of gravity at the impact site we find $0.05 \mathrm{~cm} \mathrm{~s}^{-2}$. Combining this with the volume that we have deduced we find that the bulk density of a uniform nucleus with this gravity must be $0.6 \mathrm{~g} \mathrm{~cm}^{-3}$. For a dust-to-volatile ratio of 2, assuming material densities of 2.5 (some denser silicates and some less dense organics) and 1 respectively, this implies a very high porosity of $70 \%$ for the bulk properties of the nucleus.

Prior studies of comet Shoemaker-Levy 9 (SL9) had shown us that the tensile strength at km-scale was less than $100 \mathrm{~Pa}$ (Sekanina 1996). The success of strengthless models in showing reaccretion to the size of the observed fragments makes it plausible that the strength is that low even at the 100-m scale, but does not prove it. On Earth, strength is normally higher at smaller spatial scales but our experiment suggests that this is not the case on comets since our strength is relevant to scales from 1-m to 100-m, the size of the ejecta to the size of the crater (we will argue below that the ejecta are primarily $<10 \mu \mathrm{m}$ in size). The deduced strength is even lower than that predicted by Greenberg (1995) on the basis of his model for accreting interstellar grains.

The high porosity is not surprising since studies of the non-gravitational acceleration of comets starting with the work by Rickman et al. (1987) have typically deduced bulk densities between 0.1 and $1.0 \mathrm{~g} \mathrm{~cm}^{-3}$, although the results are very model dependent as shown by the range of results for comet Wild 2 deduced by Farnham \& Cochran (2002) and by Davidson \& Gutiérrez (2004). While our own determination of the bulk density is not totally free of modelling assumptions, we think that it is much more 
tightly constrained than previous determinations. There is, thus far, no way to determine whether the porosity is primarily fine scale porosity or primarily large voids. The result assumes a uniform density, i.e. a uniform porosity but is not sensitive to that assumption unless the voids are comparable in size to our crater.

\section{The Impact Site and the Surface Layer Properties}

The impact was oblique, at $20 \mathrm{deg}-35 \mathrm{deg}$ to the horizontal. The impact site, shown in figure 2 , is between two crater-like features that are roughly $300 \mathrm{~m}$ across. The impactor approached from the top in these images, probably at about $35 \mathrm{deg}$ above the horizontal. In the last figure shown, taken $13 \mathrm{sec}$ before impact, the white spots are a few $\mathrm{m}$ in diameter. It is this terrain to which the strength measurements above apply. Whether the strength is similar in the other very different terrains that are clearly visible in the pictures of the nucleus is unknown. However, given the strength at larger scales inferred from nuclear breakup of SL9 (above) and other comets, it seems unlikely that the strength elsewhere would be dramatically different from the values deduced here. The impactor landed much closer to the lower (more southern in ecliptic coordinates, of the two craterlike features. While this area is obscured by ejecta in all post-impact images, the more northerly of the two craters is still visible after the impact with no obvious disruption. The distance from the ipact site to the southerly wall of the northerly crater thus sets an upper limit on the size of our crater at a radius of about $150 \mathrm{~m}$.

The obliquity of the impact also shows up in the successive stages of brightness as illustrated in figure 3, where three successive brightenings can be seen, each displaced from the one before it. These images are contour maps of the brightness in 50-msec exposures, taken with center-to-center intervals of $62 \mathrm{msec}$. The first flash is faint, visible in only frames 64 and 65 . This may correspond to the very early hot ejecta traveling backwards up the entry path or it may correspond to the subsurface flash of impactor destruction being scattered through the overlying layers. The second brightening, which appears after the first flash has disappeared, has the highest peak brightness, saturating the CCD in frames 67 and 68, which leads to a spatial broadening that is an artifact of charge bleeding along the column of the CCD. This brightening may correspond to the first material from the impact breaking through the surface. The final brightening, although not reaching the peak brightness of the second, is of much longer duration, beginning while the second flash is fading, and is the source of the bulk of the ejecta. The motion downrange between the successive brightenings is clear.

A sequence of images, from which the brightness profiles in figure 3 were derived, is shown in figure 4 . This sequence, numbered horizontally and then vertically, corresponds to frames 59 through 70 of figure 3, i.e., it starts earlier than and ends in the middle of the sequence in figure 3 , ending while the third brightening is still increasing. In figure 4 one can see a puff of material originating before the peak of the second brightening and rapidly moving downrange such that it is completely beyond the field of view by the end of the sequence (corresponding to frame 70). As discussed below, this is hot material driven hydronamically from the excavation which supports our interpretation of the second brightening as the first breakthrough of material at the surface of the nucleus. The horizontal row of dots in this figure shows the position of the slit of our near-infrared spectrometer during the sequence, with the bright dot in that row corresponding to the pixel that will be analyzed below. At the end of the sequence (corresponding to frame 70), the slower moving ejecta from the mechanical excavation stage have just reached the slit of the spectrograph. 


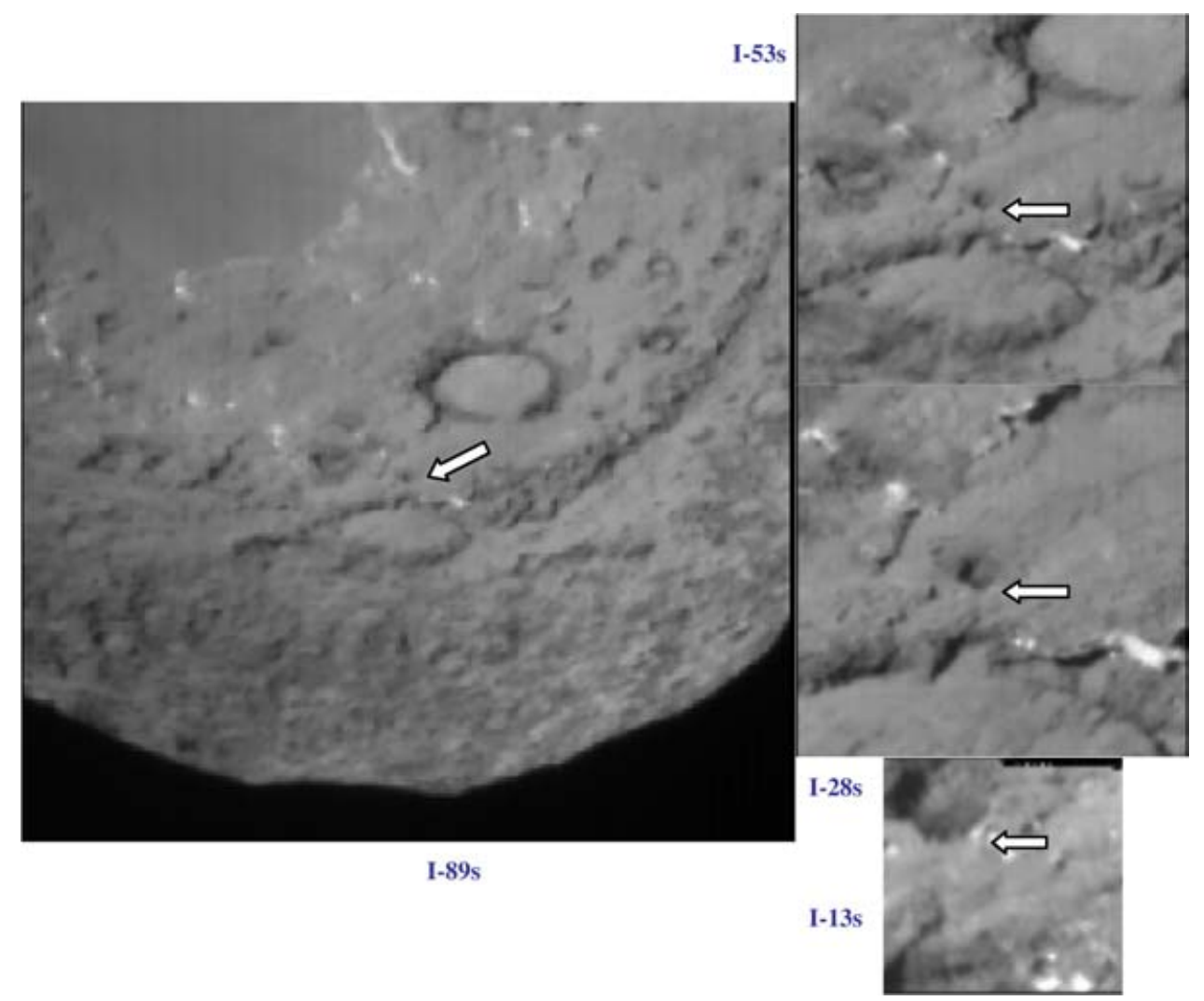

Figure 2. Successively closer images of the impact site taken by the impactor at the times shown in the figure. The ellipticity of the two craters, assuming they are both truly round, implies an approach angle of $20 \mathrm{deg}$ and $35 \mathrm{deg}$ respectively for the two craters, while the overall shape model agrees with the latter number.

\section{Ejected particles}

The particles ejected in the initial puff are very hot as measured by our near-infrared spectrometer. We obtained spectra continuously with the slit positioned as shown in figure 4 , using initially an exposure time of $0.72 \mathrm{sec}$. The first of the two spectra shown in figure 5 was exposed at the single pixel shown in the slit position and was exposed completely before the impact. It shows purely a continuous spectrum with reflection from the nucleus dominating shortward of $2.5 \mu \mathrm{m}$ and thermal emission from the nucleus dominating longward of $3.5 \mu \mathrm{m}$. At $0.5 \mathrm{sec}$ into the exposure of the second spectrum, the expanding, lateral edge of the hot puff of material reaches the chosen pixel in frame 66 (pixels closer to the centerline of the ejecta become saturated at this point) and material from the puff remains in the slit until about the end of the exposure. In order to model the second spectrum, we require not only the thermal emission from the nucleus but also a much stronger emission from material at $850 \mathrm{~K}$. Subsequently excavated material is cold and relatively unprocessed. The model for the gasous emission will be discussed below.

The emission from the hot puff has been modeled as molten, silicate droplets, although this is certainly not a unique model. The model has the droplets starting at $3500 \mathrm{~K}$ when they are ejected and cooling faster than they vaporize. The model predicts that the hot puff will be optically thin within $0.03 \mathrm{sec}$. The brightness of the puff drops rapidly, 

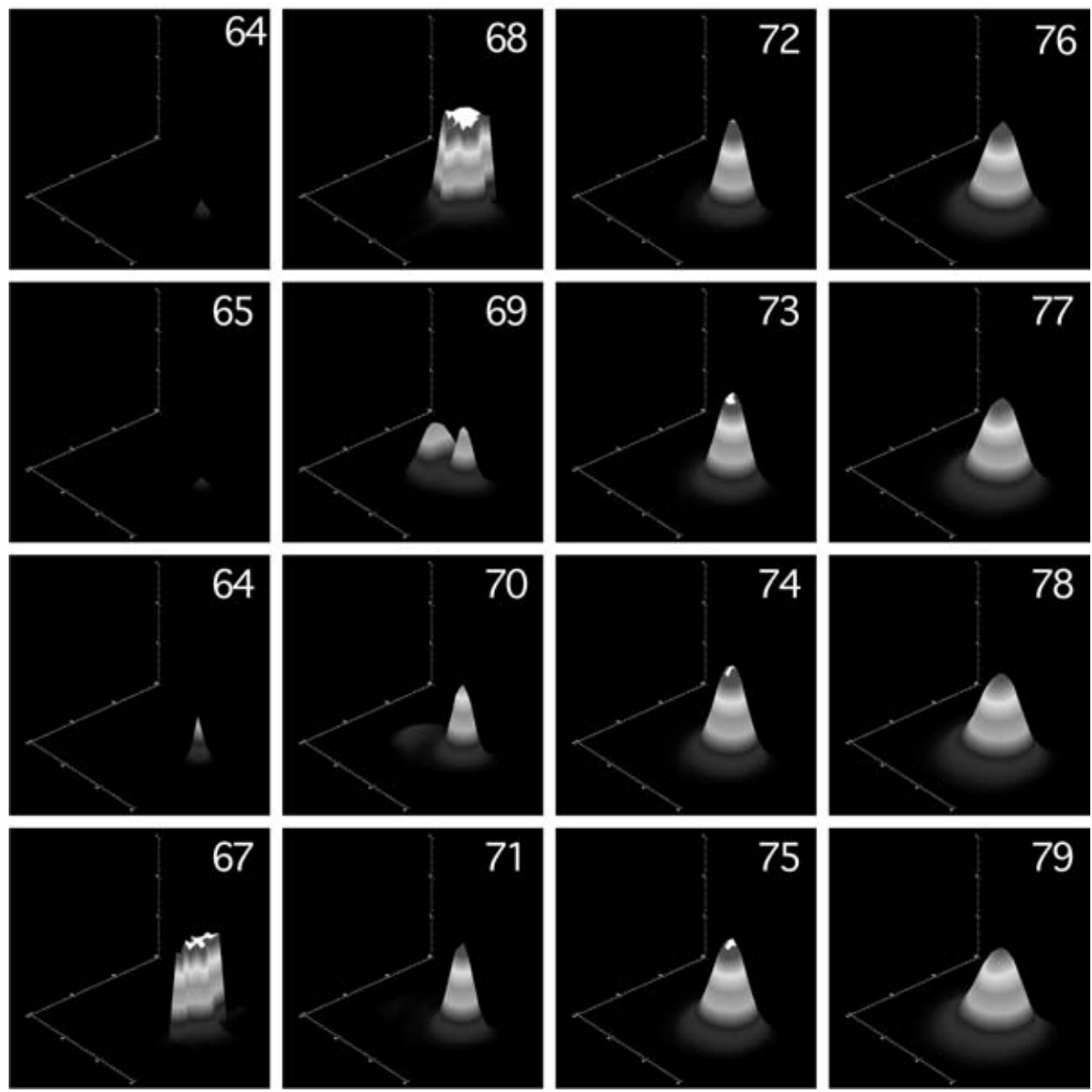

Figure 3. Brightness contours of the three successive brightenings observed at 62 -msec intervals. The 3-D perspective figures have the downrange direction, approximately north to south in ecliptic coordinates, going from upper left to lower right. Individual exposures are 50-msec each.

much too fast to be explained solely by geometric dilution. However, the combination of expansion and cooling reproduces the observed peak brightnesses very well as a function of time and is also consistent with the temperatures measured at the lateral edge of the puff. After $0.42 \mathrm{sec}$, the puff has cooled such that the brightness is dominated by reflected sunlight and the fading follows an inverse square law with time solely from geometric expansion of the cloud.

Subsequent solid ejecta, measured both in front of the nucleus very soon after impact and beyond the southern limb of the nucleus at later times, are cool with temperatures near 300 to $350 \mathrm{~K}$. This is warmer than the black-body temperature but relatively constant, suggesting that we are seeing the superheat expected from particles smaller than the wavelength of peak thermal emission. Although Keller et al. (2005) finds large particles in the ejecta when he models the radiation pressure effects for data from Rosetta, Schleicher et al. (2005) finds from a similar study of ground-based data that the particles are all small, of order $2 \mu \mathrm{m}$ or less. The resolution of the discrepancy between these two results is beyond the scope of this paper. We also note that analysis of our own 

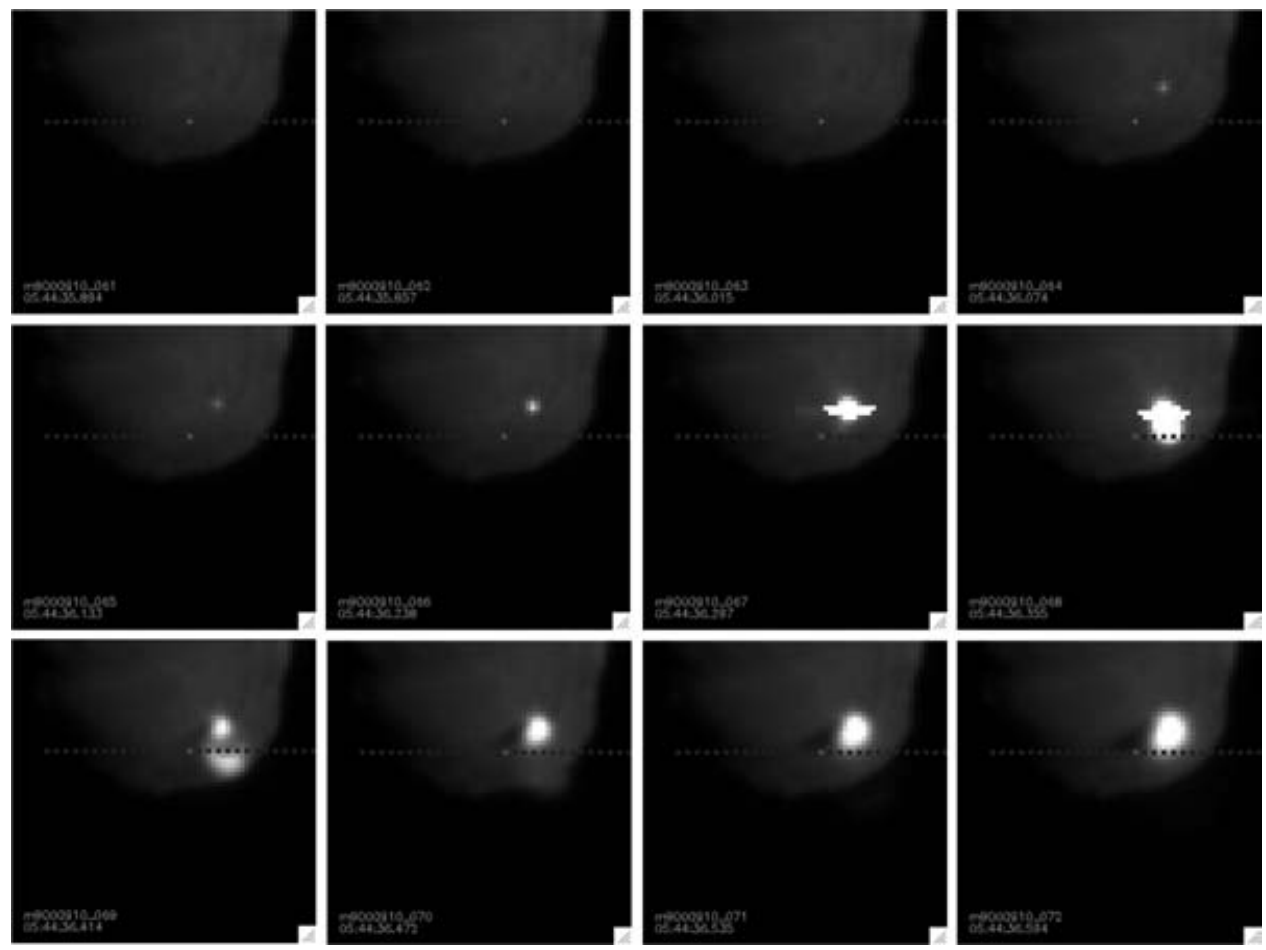

Figure 4. Sequence of images with the Medium Resolution Instrument (MRI) taken at 62-msec centers with 50-msec exposure times. Images correspond to the brightness countours of figure 3 frames 59 to 62 in the top row, 63 to 66 in the middle row, and 67 to 70 in the bottom row. A puff of rapidly moving, hot ejecta is seen in frames 65 through 69 .

data is still in progress and should cast further light on this issue. If the particles are all small, i.e., if the size distribution does not have the tail of large-mass particles common in ambient outgassing from comets, then we must conclude that the material prior to excavation was already in these small particles or in weak aggregates thereof. The latter makes more sense, i.e. that particles seen as large in remote sensing data are actually very weak aggregates of smaller particles. Presumably the mechanical excavation disrupted these aggregates more than does the gas drag of ambient outgassing.

The cone of ejected particles is optically thick at all times from impact until the flyby spacecraft flew past the nucleus some 14 minutes after the impact. The cone casts a prominent shadow on the nucleus as soon as 2 seconds after impact and the depth of the shadow requires an optical depth of at least a few. Meausrements of the contrast at the limb more than 10 minutes after impact yield an optical depth between 2 and 3 . These results all point to the ejected mass being confined to very small particles.

\section{Volatiles Released}

Figure 5 shows gaseous emissions in addition to continua from reflected sunlight and thermal emission. A model has been fitted to the observed spectrum using water, hydrogen cyanide, and carbon dioxide. This is shown as the gray line in the figure. The model did not include organics, which are also obvious in the observed spectrum via the $\mathrm{C}-\mathrm{H}$ stretch feature that is common to many organics over a range of wavelengths centered at $3.4 \mu \mathrm{m}$. The three gaseous species were all at $1400 \mathrm{~K}$, but any temperature between 


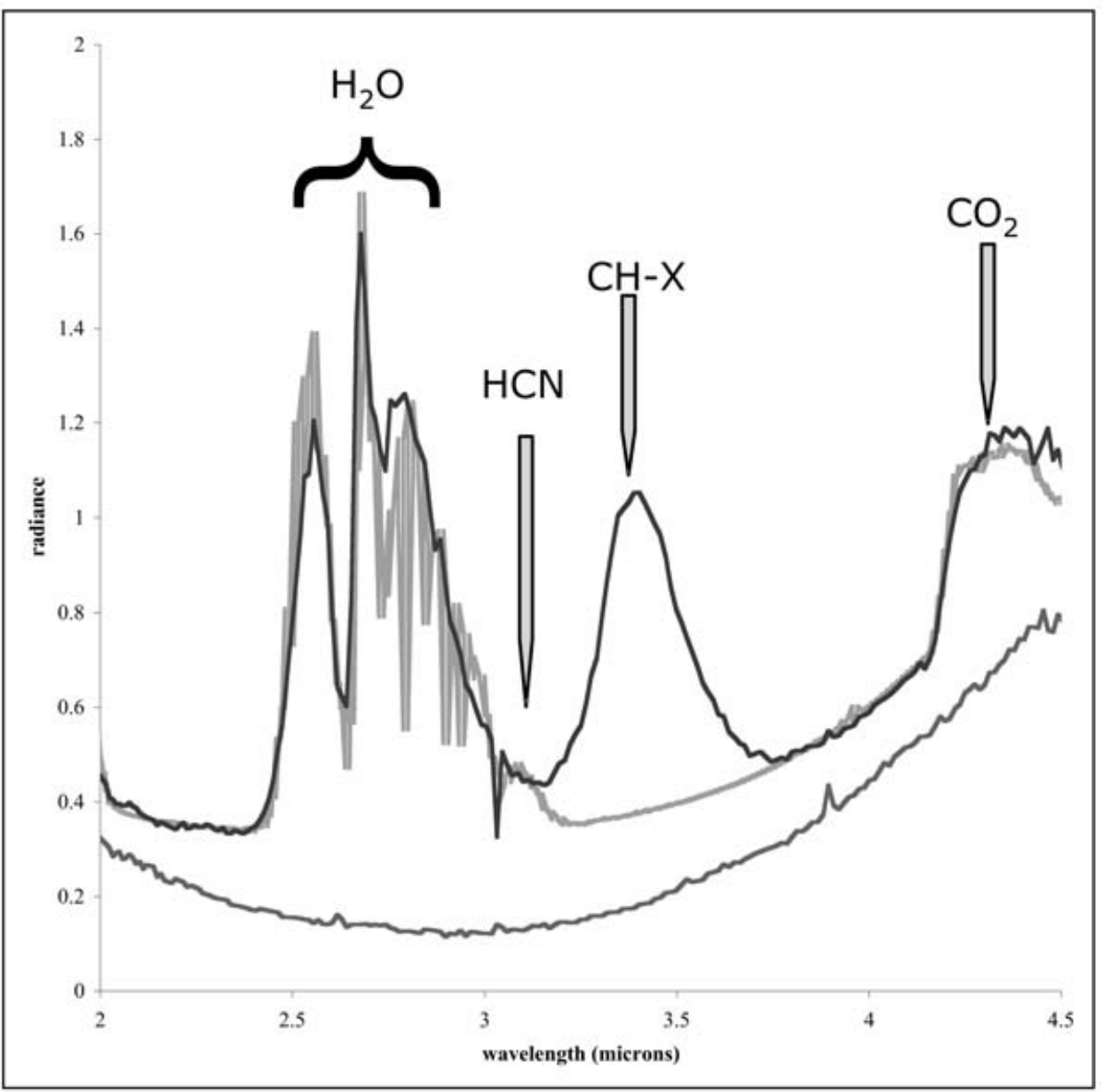

Figure 5. Two spectra, the lowest curve being the observed spectrum immediately before impact and the smooth upper curve being obtained as the puff of hot material passed in front of the slit. The upper spectrum has been fit with a model described in the text.

1000 and $2000 \mathrm{~K}$ provides a satisfactory fit. The details of the fit are not valid because the individual lines appear to be optically thick but the general trend of hot material in the puff followed by cooler material in later spectra is in excellent agreement with the changes seen in the continuum.

Spectra of the coma beyond the southern limb before and after impact are shown in figure 6 . The two spectra are at different scales of intensity by a factor 6 in order to adequately display the structure in both. The increase in all emission features is dramatic. The biggest change in relative abundances is a large increase in the ratio of organics to water. In addition, the organic feature has become much broader and contains much more structure after impact than before. This indicates many new species that were below the detection limit prior to impact. We suggest that one of these species may be methyl cyanide $\left(\mathrm{CH}_{3} \mathrm{CN}\right)$, for which at least two peaks are coincident with expected peaks, although we realize that this may be controversial since it would imply rather high abundances. Detailed analysis of the gaseous spectra will take a very long time. 

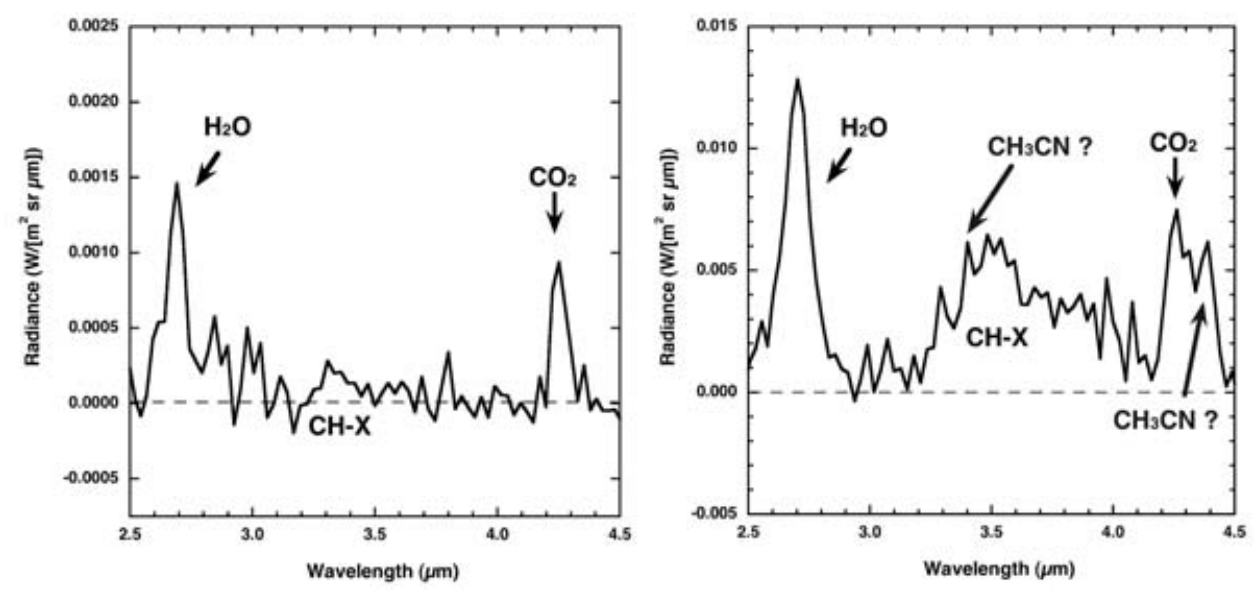

Figure 6. Spectra of the coma beyond the southern limb before (left) and after (right) impact.

\section{Summary}

The key conclusions from our impact are that the cometary material is extremely weak in the outer layers and that it consists of very weak aggregates of particles small compared to thermal wavelengths. The bulk gravity of the nucleus suggests a porosity well in excess of $50 \%$. The initial excavation led to a huge increase in organics relative to $\mathrm{H}_{2} \mathrm{O}$ and a smaller increase in $\mathrm{CO}_{2}$ relative to $\mathrm{H}_{2} \mathrm{O}$. The existence of new species is clear but the identification is not.

\section{Acknowledgements}

This project was funded by NASA, as the eighth mission in the Discovery Program, through a contract to the University of Maryland and a task order to the Jet Propulsion Laboratory.

\section{References}

A'Hearn, M.F., Belton, M.J.S., Delamere, A. \& Blume, W.H. 2005, Space Sci Rev 117, 1-21

A'Hearn, M.F., Belton, M.J.S., Delamere, W.A., et al. 30, 2005, Science 310, 265-269

Davidsson, B.J.R. \& Gutiérrez, P. J. 2004, Icarus 168, 392

Greenberg, R. 1995, Icarus 10, 20

Farnham, T.L. \& Cochran, A.L. 2002, Icarus 160, 398

Keller, H.U., Jorda, L. Küppers, M., et al. 2005 Nature 310, 281-283

Richardson, J.E., Melosh, H.J., Artemeiva, N.A. \& Pierazzo, E. 2005, Space Sci Rev 117, 241-267

Rickman, H., Kamel, L. Festou, M.C. \& Froeschle, C. 1987 in: E.J. Rolfe \& B. Battrick (eds.), Diversity and Similarity of Comets, ESA SP278, (Noordwijk: European Space Agency), p. 471-481

Schleicher, D.G., Barnes, K.L. \& Baugh, N.F. 2005, Astron. J. submitted

Schultz, P.H., Ernst, C.M. \& Anderson, J.L.B. 2005, Space Sci Rev 117, 207-239

Sekanina, Z.Z. 1996, in: K.S. Noll, H.A. Weaver \& P.D. Feldman (eds.), The Collision of Comet Shoemaker-Levy 9 with Jupiter (Cambridge: Cambridge Univ. Press), p. 55. 